\title{
Tendencias de la mortalidad por cáncer de mama en México, 1980-2009
}

\author{
Elvia de la Vara-Salazar, MEP, (1) Leticia Suárez-López, MD, (1) Angélica Ángeles-Llerenas, MC, ${ }^{(2)}$ \\ Gabriela Torres-Mejía, D Sc, ${ }^{(2)}$ Eduardo Lazcano-Ponce, D Sc. ${ }^{(3)}$
}

\author{
De la Vara-Salazar E, Suárez-López L, Ángeles-Llerenas A, \\ Torres-Mejía G, Lazcano-Ponce E. \\ Tendencias de la mortalidad por cáncer \\ de mama en México, 1980-2009. \\ Salud Publica Mex 20I I;53:385-393.
}

\section{Resumen}

El cáncer de mama ha trascendido como un importante riesgo para la salud de las mujeres a escala mundial. Las muertes por cáncer de mama han tenido un destacado incremento dentro de los tumores malignos a nivel mundial al cobrar más de 460000 vidas en 2008, convirtiéndolo en el padecimiento con más decesos alrededor del mundo. Los cambios demográficos y en estilos de vida han modificado la exposición de la población al riesgo de enfermedades como el cáncer,y desde 1980 la mortalidad por cáncer de mama ha mantenido una tendencia ascendente, ubicándose por encima de las muertes por cáncer cervicouterino desde 2006. Al analizar las tasas de mortalidad en las mujeres mexicanas de 25 años de edad en adelante a lo largo de 30 años, se distinguen diferencias estatales y por grupos de edad. Aun cuando el perfil de esta causa de muerte se ha asociado con un mayor desarrollo regional, en este trabajo se puede observar que están ocurriendo cambios y las muertes también están creciendo en la población de mujeres de regiones y entidades menos desarrolladas. Queda de manifiesto el reto que México enfrenta, y la necesidad de conjuntar esfuerzos e implementar programas para educar a la población hacia el autocuidado de la salud, así como promover estilos de vida saludables, además de mejorar la infraestructura diagnóstica para lograr una detección a tiempo y garantizar un tratamiento adecuado.

Palabras clave: neoplasias de la mama; mortalidad; México
De la Vara-Salazar E, Suárez-López L, Ángeles-Llerenas A, Torres-Mejía G, Lazcano-Ponce E. Breast cancer mortality trends in Mexico, 1980-2009.

Salud Publica Mex 20I I;53:385-393.

\begin{abstract}
Breast cancer has become an important health risk for women worldwide.The important growth of breast cancer-related deaths within those caused by malign tumors throughout the globe went past the 460000 in 2008, becoming the deadliest disease worldwide. Demographic changes and lifestyles have modified the population exposure to risk factors of maladies such as cancer, and since 1980 breast cancer mortality has remained on an upward tendency, surpassing cervical cancer in 2006. After analyzing mortality rates along 30 years in Mexican women 25 or more years old, differences by state and age-groups are apparent. Although this cause of death has been associated with a highest regional development, some changes are taking place, since the number of deaths is also growing among women of less-developed regions in the country, as showed in this work. Mexico faces an evident challenge regarding breast cancer. Our country requires to join efforts and implement programs aimed at teaching self-care of health among the population, promoting healthier lifestyles, and reshaping our diagnostic infrastructure to achieve earlier detection and provide proper treatment.
\end{abstract}

Keywords: breast neoplasms; mortality; Mexico

(I) Dirección de Salud Reproductiva, Centro de Investigación en Salud Poblacional, Instituto Nacional de Salud Pública. México.

(2) Dirección de Enfermedades Crónicas, Centro de Investigación en Salud Poblacional, Instituto Nacional de Salud Pública. México.

(3) Centro de Investigación en Salud Poblacional, Instituto Nacional de Salud Pública. México. 
E cáncer de mama ha tenido una trayectoria ascendente en las últimas décadas, que se manifiesta con el incremento de las muertes por esta causa entre mujeres de 25 años de edad en adelante. Esta situación se vincula con los estilos de vida, así como con las transiciones demográfica y epidemiológica, procesos que han favorecido el aumento en la esperanza de vida de la población y por ende el desarrollo de enfermedades crónico-degenerativas. ${ }^{1,2}$

El cáncer se encuentra entre los padecimientos que destacan en el panorama epidemiológico mundial, cuya tasa de incidencia y mortalidad varía en relación con el área geográfica y las condiciones de vida. ${ }^{3}$ Éste se ubica como la principal causa de mortalidad en el mundo con 7.6 millones de defunciones (aproximadamente 13\% del total ocurridas en 2008); de éstas, 460000 se debieron a fallecimientos por cáncer de mama, lo que lo sitúa entre los tipos de cáncer que cobran más vidas alrededor del mundo. ${ }^{4}$

El cáncer de mama es el más común entre las mujeres a nivel mundial, pues representa $16 \%$ de todos los cánceres femeninos $\mathrm{y}$, aunque este padecimiento está considerado como una enfermedad del mundo desarrollado, la mayoría de las defunciones por esta causa se registran en los países en desarrollo, ${ }^{5,6}$ con variaciones importantes al interior de éstos. El aumento de la incidencia de cáncer de mama en el mundo en desarrollo se relaciona con una mayor esperanza de vida de la población, el progreso de la urbanización y la adopción de modos de vida occidentales, aunado a la deficiente detección precoz para mejorar el pronóstico y la supervivencia de estos casos. Las bajas tasas de supervivencia observadas en estos países pueden explicarse principalmente por la falta de programas de detección precoz, que hace que un alto porcentaje de mujeres acudan al médico con la enfermedad ya muy avanzada, pero también por la falta de servicios adecuados de diagnóstico y tratamiento. ${ }^{2}$ Además, la creciente adopción de estilos de vida menos saludables en los países de ingresos bajos y medios es un determinante importante del incremento de la incidencia de cáncer de mama en esos países.

Aunque se conocen varios factores de riesgo del cáncer de mama, en la mayoría de las mujeres afectadas no es posible identificarlos específicamente; ;,8 la incidencia diferencial del cáncer de mama en los países puede explicarse además por los efectos de la alimentación, unidos a la mayor edad al primer embarazo, el menor número de partos y el acortamiento de la lactancia. ${ }^{9}$

La Organización Mundial de la Salud promueve la lucha contra el cáncer de mama dentro del marco general de programas nacionales de control del cáncer que están integrados con las enfermedades no transmisibles y otros problemas relacionados. El control integral del cáncer abarca la prevención, la detección precoz, el diagnóstico y tratamiento, la rehabilitación y los cuidados paliativos.

En México, de las más de 434000 defunciones generales registradas en 1980 en todo el país, 45.4\% se debieron a enfermedades no transmisibles, ${ }^{10} \mathrm{y}$ para 2008 , de las 539500 defunciones, $75.4 \%$ fueron causadas por estas mismas enfermedades, lo que significa un incremento de 30 puntos porcentuales durante el periodo. ${ }^{2}$

La situación de la mortalidad por cáncer en México es considerada como un problema prioritario de salud pública, ya que desde 1990 los tumores malignos representan la segunda causa de muerte en la población general y su ascenso ha sido sostenido. Hasta la década de los setenta, México era de los países latinoamericanos con tasas de mortalidad más bajas, ${ }^{11}$ sin embargo, desde 1979 ha tenido una tendencia ascendente, ${ }^{1,12}$ que alcanzó a poner a esta enfermedad en la cima de las muertes por cáncer a partir de 2006, con importantes variaciones entre las entidades del país.

El destacado incremento de la mortalidad por cáncer de mama -dentro de los tumores malignos- en las mujeres mexicanas ha sido constante desde hace varias décadas de acuerdo con el número de defunciones y las tasas de mortalidad registradas; esta situación ha constituido una prioridad para países como el nuestro, y ha sido abordada en la literatura médica como un problema de salud pública, pues a partir de 2006 estas muertes superaron las causadas por cáncer cervicouterino. ${ }^{12,13}$

La evidencia observada en las defunciones femeninas por cáncer de mama en la población nacional presenta un registro de 1206 muertes en 1980, que alcanza un total de 4897 en 2009; dichos valores absolutos representan 0.3 y $0.9 \%$ respecto de las defunciones generales nacionales de esos años.

Diversos estudios epidemiológicos, entre los ya referidos, mencionan que el cáncer de mama está asociado con factores ambientales entre los que se encuentran el estado nutricio, la exposición a pesticidas, el consumo abundante de grasa animal y no haber amamantado, ${ }^{3}$ aunque estos factores aún generan controversia sobre su posible participación en la presentación de este padecimiento. Dadas las características antes mencionadas, aunadas al nivel socioeconómico de la población, ciertas prácticas alimenticias y bajos hábitos de lactancia, es posible suponer que la distribución de esta neoplasia en México puede variar debido a la heterogeneidad del entorno sociocultural y económico de las entidades y regiones. Como ejemplo se tiene que en los estados del norte la población tiene ingresos económicos más elevados, y se consume una dieta que favorece el sobrepeso y la obesidad. De acuerdo con las estadísticas de 
mortalidad por cáncer de mama en 2002, ésta fue más elevada en los estados que se ubican al norte del país, así como en el Distrito Federal y Jalisco. ${ }^{3}$

El objetivo general de este trabajo es describir la tendencia de la mortalidad por cáncer de mama en las mujeres mexicanas de 25 años de edad en adelante, de 1980 a 2009, por entidad de residencia y por grupos quinquenales de edad.

La descripción y tendencia de la mortalidad por cáncer de mama se analiza a través de tasas estandarizadas. La información básica para el cálculo de las tasas proviene de las defunciones registradas en las estadísticas vitales, disponibles en línea en la base de datos de defunciones 1979-2009 del Sistema Nacional de Información en Salud (SINAIS). ${ }^{14}$ Con la consulta de la base de defunciones se construyeron las "tablas dinámicas" de acuerdo con la clasificación de "principales causas" de la lista del Global Burden of Disease (GBD), a nivel nacional y por entidad federativa, y se seleccionaron las características de interés: defunciones por cáncer de mama, población femenina por grupos de edad de 25 años en adelante y entidad de residencia, y se excluyeron los casos "no especificados". Los datos de la población media femenina a nivel nacional y por entidad federativa, desagregados por edad, provienen de las proyecciones de población 1980-2030 del Consejo Nacional de Población (CONAPO). ${ }^{15}$

La población femenina estándar se tomó de la revisión del 2008 de Naciones Unidas, ${ }^{16}$ la cual sólo está disponible cada cinco años, por lo que fue necesario calcular la población para los años intermedios (1981$1984,1986-1989, \ldots$ etc.), por grupos quinquenales de edad de 25 años en adelante.
Para analizar la información se calcularon las tasas de mortalidad por edad y por entidad federativa, y con fines de comparación se efectuó el procedimiento de estandarización directa, en el que se estima el valor que tomaría la tasa de mortalidad cuando se "elimina" el efecto que ejerce sobre esta medida la estructura por edad de la población. ${ }^{17}$ Para el cálculo de estas tasas a nivel nacional, se tomó como referencia la estructura por edad de la población femenina mundial de Naciones Unidas, ${ }^{16}$ y para las tasas por entidad federativa, se utilizó como población de referencia la estructura por edad de la población femenina nacional. ${ }^{15}$

\section{Escenario de la mortalidad por cáncer de mama}

El cáncer de mama presenta una tendencia ascendente en el periodo analizado, la cual se muestra con las defunciones y las tasas de mortalidad estandarizadas.La distribución de estas defunciones por cáncer de mama presenta una tendencia hacia el incremento, la cual ha sido paulatina pero sostenida desde 1980, al pasar de 1199 defunciones a 4893 en 2009, de mujeres de 25 años de edad en adelante (figura 1). Lo que es un hecho inaceptable son las 86469 muertes causadas por esta enfermedad en los últimos 30 años.

\section{Evolución de la mortalidad por cáncer de mama}

La tendencia ascendente de la mortalidad por cáncer de mama en el periodo observado es evidente, al pasar de una tasa estandarizada de 11.7 fallecidas por cada 100000 mujeres en 1980 a 17.0 en 2009. Sólo se observan

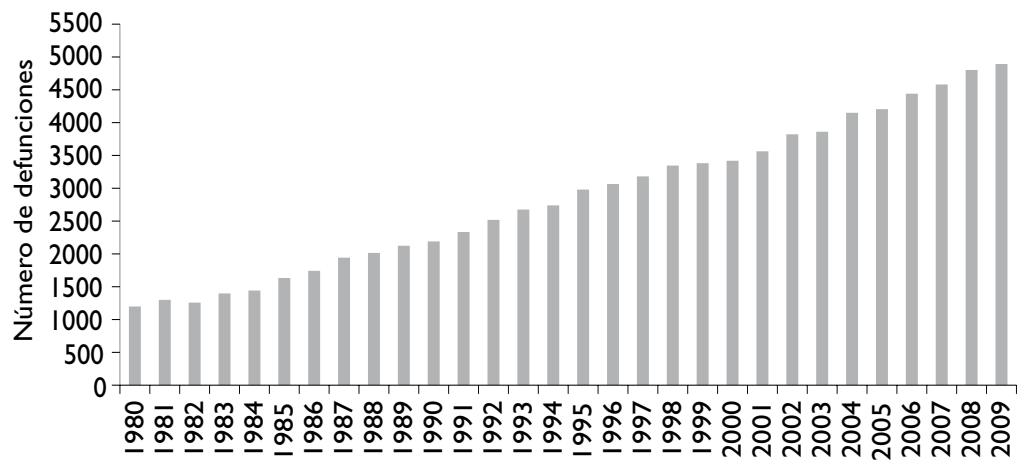

- Defunciones por cáncer de mama en mujeres de 25 años en adelante

Fuente: Cálculos propios con base en las proyecciones de población de CONAPO, 1980-2030 y en la Base de datos de defunciones, 1979-2009, SINAIS

Figura I. Número de defunciones por cáncer de mama en México, 1980-2009 
leves caídas en 1982 (11.5) y 2000 (15.6), que se revirtieron al siguiente año (figura 2).

La tendencia observada de las tasas de mortalidad estandarizadas observadas por década muestra que pasaron de 11.7 a 14.7 en los años ochenta, mientras que en la década de los noventa se incrementaron de 14.6 a 16.0, y en los últimos diez años de 15.6 a 17.0 muertes por 100000 mujeres de 25 años en adelante.

Al analizar la información de defunciones por grupos de edad se observan porcentajes más elevados en las mujeres de 40 a 64 años, con tendencia ascendente durante el periodo, mientras que en las mujeres de 65 años en adelante las defunciones disminuyen respecto de los grupos de edad anteriores; sin embargo,la tendencia en el tiempo no declina, inclusive de 2005 a 2009, pues se presenta un leve incremento en el grupo de mujeres de 75 años de edad en adelante (figura 2).

\section{Cáncer de mama y mortalidad por grupos de edad}

Las tasas de mortalidad por cáncer de mama desagregadas por edad quinquenal permiten una visión más fina del indicador. De manera general se pude observar que la tendencia de la mortalidad por grupos de edad tiene concordancia con la nacional.

La tasa más elevada se ubica en el grupo de mujeres de 75 años en adelante, al pasar de 38.8 en 1980 a alcanzar el valor más alto (62.0) del periodo en 1998, y a partir de 1999 se mantuvo hasta 2008, año en que descendió a 58.9, y a 57.9 en 2009 (figura 3). En los grupos de 55 a 74 años de edad se presenta la misma tendencia que en el grupo anterior, con tasas que se incrementan. En cambio, en los grupos de edad de 35 a 54, aunque también existe una tendencia ascendente, a partir del 2000 tiende a decrecer.

El perfil de la mortalidad por cáncer de mama en los dos grupos más jóvenes es distinto, dado que las tasas se han mantenido relativamente estables durante el periodo, en comparación con los grupos de mayor edad; sin embargo, las tasas no dejan de ser relevantes, sobre todo en los grupos de 35 a 44 años de edad (figura 3). De acuerdo con estudios previos en México, el incremento de las tasas de mortalidad se atribuye, entre otros factores, a los cambios en los estilos de vida que se acercan a los de la población de países más desarrollados. ${ }^{3,9}$

Las diferencias en la tendencia de mortalidad por grupos de edad (figura 3) quizás se deban a la existencia de un mayor conocimiento de la enfermedad y a la disponibilidad de servicios para el diagnóstico y el tratamiento oportunos, sobre todo entre los grupos más jóvenes.

Para observar el cambio que las tasas de mortalidad han tenido en el tiempo estudiado, se observa el porcentaje que representa el incremento de las tasas de mortalidad por cáncer de mama por grupos de edad, el cual se muestra en periodos de cinco años (cuadro I), donde se observa un importante incremento en 19851989 respecto de 1980-1984 en todos los grupos de edad, excepto en el grupo de mujeres de 25 a 29 años, donde hubo un decremento de 14 por ciento. En el siguiente quinquenio, el porcentaje de incremento de las tasas de mortalidad disminuyó en la mayoría de los grupos de edad respecto del anterior, excepto en los grupos de edad de 25-29 y 60-69, que alcanzaron un porcentaje de 17.3 y 8.7\%, respectivamente. De 1995-1999 a 2000-2004 en todos los grupos de edad el porcentaje de cambio de

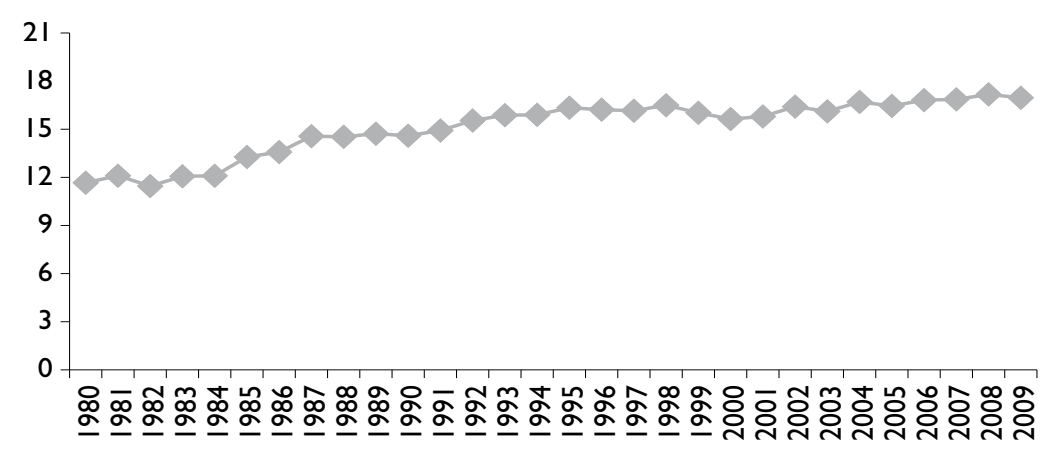

_ Tasa de mortalidad estandarizada por cáncer de mama

Tasa por 100000 mujeres de 25 años en adelante

Fuente: Cálculos propios con base en las proyecciones de población de CONAPO, 1980-2030 y en la Base de datos de defunciones, I979-2009, SINAIS

Figura 2.TASAs de mortalidad estandarizadas por CÁnCer de mama en MéXico, 1980-2009 

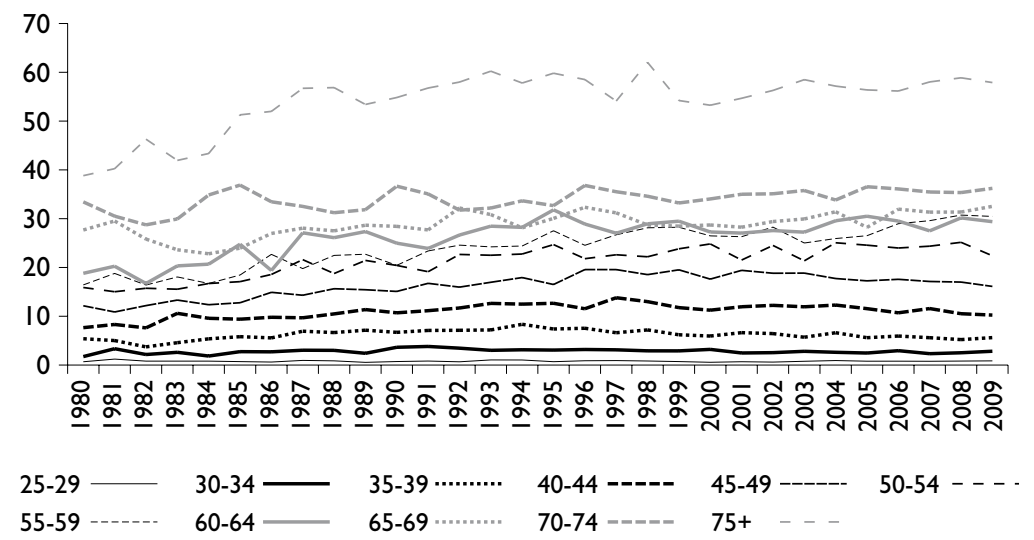

Tasa por 100000 mujeres de 25 años en adelante

Fuente: Cálculos propios con base en las proyecciones de población de CONAPO, 1980-2030 y en la Base de datos de defunciones, 1979-2009, SINAIS

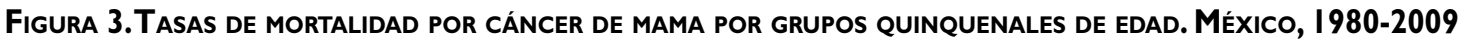

Cuadro I

Porcentaje de cambio de las tasas de mortalidad por CÁncer de mama, POR GRUPOS QUINQUENALES DE EDAD DE I980-I984 A 2005-2009. MÉXICO

\begin{tabular}{|c|c|c|c|c|c|}
\hline Quinquenios/ & de 1980-1984 & de 1985-1989 & de 1990-1994 & de 1995-1999 & de 2000-2004 \\
\hline Edad & a 1985-1989 & a 1990-1994 & a 1995-1999 & a 2000-2004 & a 2005-2009 \\
\hline 25 a 29 & -14.3 & 17.3 & -6.8 & -12.0 & 13.7 \\
\hline 30 a 34 & 19.0 & 22.3 & -10.7 & -10.2 & -4.0 \\
\hline 35 a 39 & 33.9 & 12.6 & -4.0 & -10.0 & -10.8 \\
\hline 40 a 44 & 15.7 & 15.6 & 6.6 & -4.8 & -8.8 \\
\hline 45 a 49 & 20.2 & 13.2 & 13.2 & -1.6 & -8.0 \\
\hline 50 a 54 & 23.7 & 10.3 & 6.8 & 1.9 & 2.6 \\
\hline 55 a 59 & 23.1 & 10.3 & 15.4 & -2.5 & 11.2 \\
\hline 60 a 64 & 29.0 & 5.9 & 10.2 & -4.9 & 5.8 \\
\hline 65 a 69 & 5.2 & 8.7 & 2.1 & -1.7 & 5.2 \\
\hline 70 a 74 & 5.0 & 2.1 & 2.3 & 0.6 & 3.4 \\
\hline $75+$ & 28.2 & 6.4 & 0.1 & -2.7 & 2.6 \\
\hline
\end{tabular}

Fuente: Cálculos propios con base en las proyecciones de población de CONAPO, 1980-2030 y en la Base de datos de defunciones, 1979-2009, SINAIS

las tasas de mortalidad disminuyó, excepto en los grupos de edad de 70-74 y 50-54; sin embargo, en el último periodo de 2000-2004 a 2005-2009 el incremento es más claro en los grupos de mujeres de 50 años en adelante, al presentar porcentajes más elevados que en el periodo anterior, así como un descenso continuado en las tasas de mortalidad de los grupos más jóvenes, a excepción del de 25 a 29 años de edad, donde hubo un incremento de 13.7 por ciento.
Diferencias estatales de la mortalidad por cáncer de mama

La distribución por entidad federativa de la mortalidad por cáncer de mama en el año 1980 muestra el nivel más alto de las tasas en los estados de la franja fronteriza norte, que va de 9.5 a 20.2, entre los que destacan Baja California, Baja California Sur, Sonora, Chihuahua, Coahuila, Nuevo León, Tamaulipas, así como en Sinaloa, Aguascalientes, Jalisco, Colima y el Distrito Federal 
(figura 4). En este grupo la entidad con la tasa más alta es Baja California, con 20.2 muertes por cada 100000 mujeres, y en contraste está Aguascalientes con la más baja (12.6). En el nivel de tasas intermedio, que va de 5.2 a 9.5, se ubican los estados de Durango, Estado de México, Hidalgo, Michoacán, Morelos, Nayarit, Puebla, Querétaro, San Luis Potosí, Tabasco, Veracruz y Yucatán; en este grupo, Tabasco posee la tasa más baja (5.5), y la más alta la tienen Michoacán y Morelos (9.4). En el nivel de tasas de mortalidad bajo (1.8 a 5.2) están la mayoría de los estados del sureste y otros del centro norte del país, como Zacatecas; la tasa más baja la tiene Guerrero con 1.8, y la más alta Guanajuato con 5.2. Entre las entidades que conforman los estados del norte resalta Zacatecas con la tasa más baja (4.5). Del total de las entidades del país, Baja California registró la tasa más elevada (20.2) y Guerrero las más baja (1.8) en 1980. Los resultados mostrados evidencian una mayor heterogeneidad de las tasas en las entidades del nivel alto y medio, mientras que entre las tasas de las entidades con nivel bajo se observa menor variabilidad.
Las tasas de mortalidad de 2009 en las entidades del país se modificaron respecto a 1980 al presentar incrementos importantes (figura 5). En la distribución geográfica de la mortalidad por cáncer de mama para este año, la mayoría de los estados del norte continúan en el nivel de tasas altas que va de 17.7 a 26.7 (Baja California, Baja California Sur, Chihuahua, Coahuila, Nuevo León, Tamaulipas, y además Colima, Jalisco, Querétaro y Michoacán), aunque con distintos niveles respecto de 1980. En este grupo la tasa más alta la sigue teniendo Baja California con 26.7 muertes por cada $100000 \mathrm{mu}-$ jeres, y la más baja la tiene Michoacán con 19.0. (figura 5). En el nivel medio, con tasas que van de 13.0 a 17.8, están los estados de Sonora, Tlaxcala, Aguascalientes, Sinaloa, Nayarit, Guanajuato, Distrito Federal, San Luis Potosí, Hidalgo, Tabasco, Puebla y Zacatecas; dentro de este grupo, Sonora posee la tasa más alta (17.8) y Zacatecas la más baja (13.6). En el nivel más bajo (9.1 a 13.0) de tasas de mortalidad están la mayoría de los estados del sureste: Chiapas, Quintana Roo, Morelos, Veracruz, Yucatán, Durango, Guerrero, Estado de México, Cam-

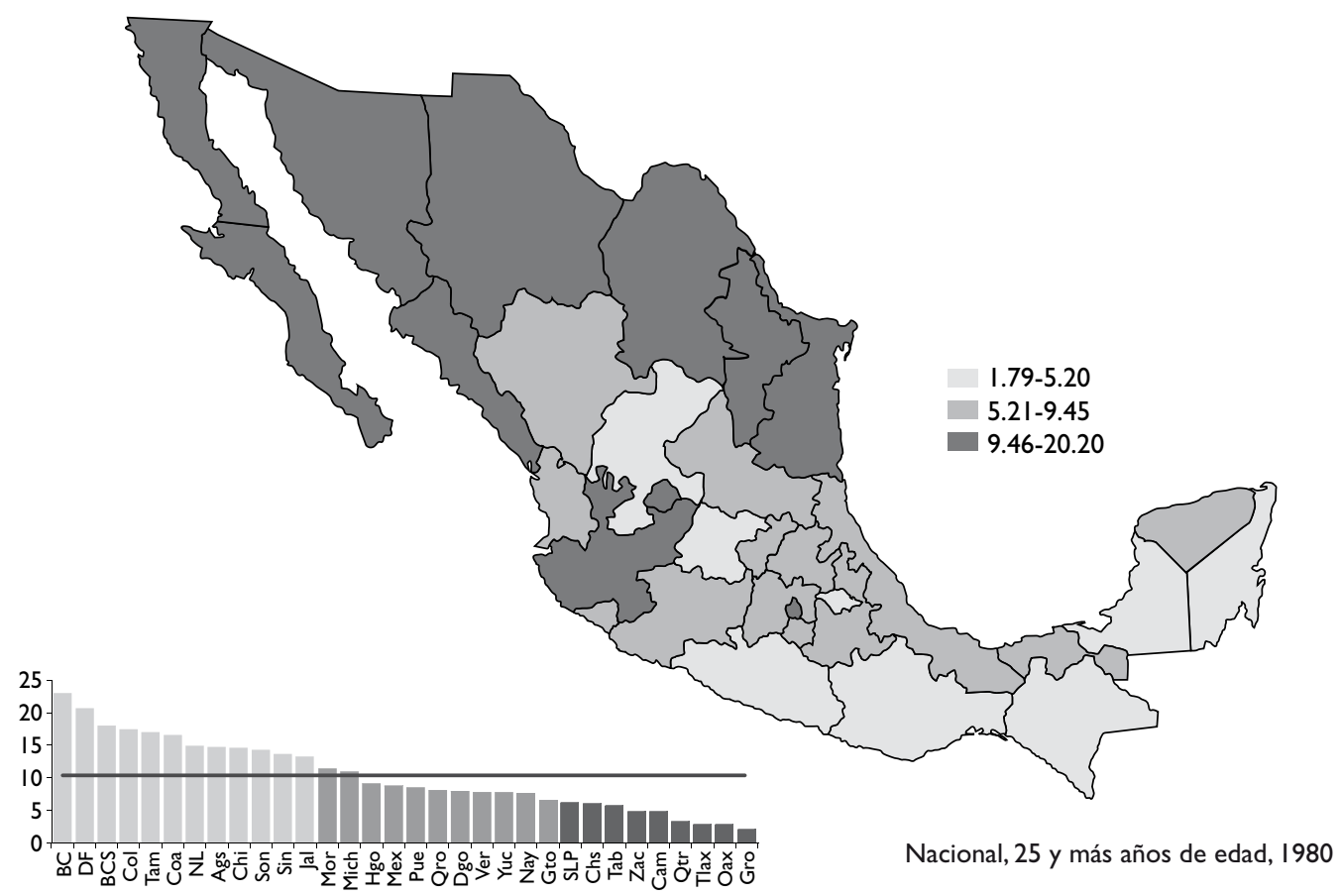

Tasa por 100000 mujeres de 25 años en adelante

Fuente: Cálculos propios con base en las proyecciones de población de CONAPO, 1980-2030 y en la Base de datos de defunciones, I979-2009, SINAIS Mapa elaborado por el equipo de trabajo de la Subdirección de Geografía Médica y Sistemas del Centro de Información para Decisiones en Salud Pública

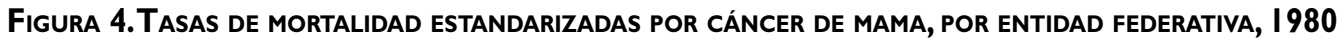




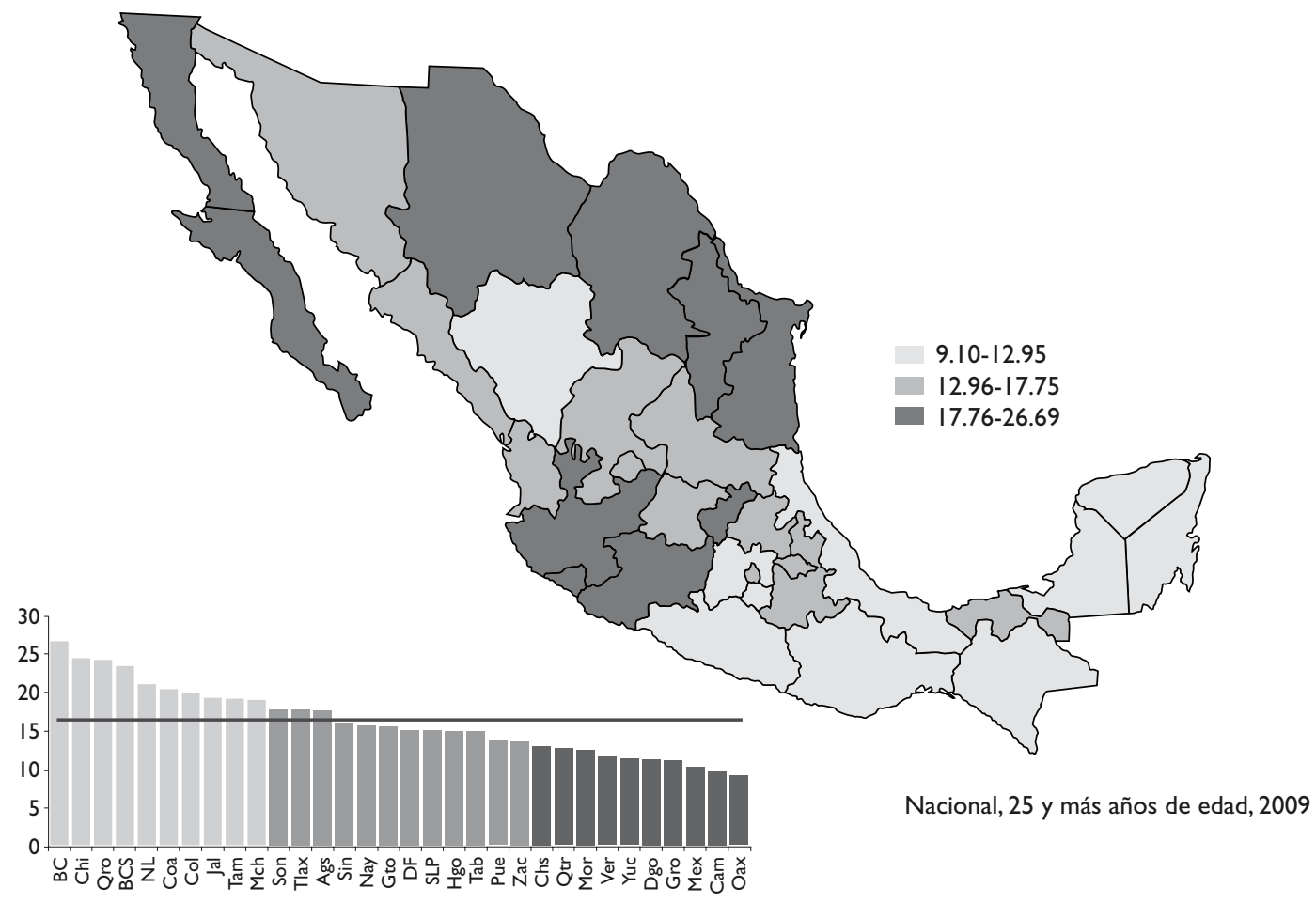

Tasa por 100000 mujeres de 25 años en adelante

Fuente: Cálculos propios con base en las proyecciones de población de CONAPO, 1980-2030 y en la Base de datos de defunciones, 1979-2009, SINAIS Mapa elaborado por el equipo de trabajo de la Subdirección de Geografía Médica y Sistemas del Centro de Información para Decisiones en Salud Pública

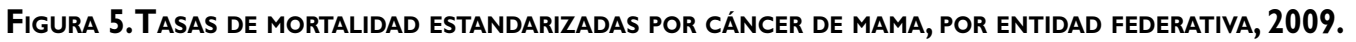

peche y Oaxaca; la tasa más alta la tiene Chiapas (13.0) y la más baja Oaxaca (9.1). Del total de las entidades del país, Baja California se mantiene con la tasa más elevada (26.7) y Oaxaca con la más baja (9.1) en 2009.

En este año se observa una mayor heterogeneidad de las tasas en las entidades del nivel alto; en las entidades con tasas de nivel medio y bajo se redujo la brecha de variación.

La evidencia de los datos muestra una distribución modificada de la geografía y de los niveles de las tasas respecto de 1980, en donde se observa la incorporación de nuevas entidades con tasas más elevadas. Algo destacable es el incremento que tienen las tasas en las entidades mexicanas en 2009 respecto de 1980; tal es el caso de Baja California, que en 1980 tuvo una tasa de mortalidad de 20.2 y para 2009 se incrementó a 26.7, y de Guerrero, que en 1980 tenía una tasa de 1.8 y para 2009 es de 11.1.

\section{Discusión}

El desarrollo económico de los últimos sesenta años en el país, aunado a las tendencias de los fenómenos demográficos como la mortalidad, trajo consigo un proceso de modernización y urbanización que benefició las condiciones de vida de la población y, por ende, se modificó el comportamiento y el estilo de vida. Estos cambios influyeron en la transformación del panorama epidemiológico de la población, de tal manera que ahora las enfermedades crónico-degenerativas, como el cáncer de mama, se han convertido en un problema importante de salud pública, y es la primera causa de muerte por tumores malignos de mujeres en México desde 2006. $13, *$

* Lozano R, Knaul FM, Gómez-Dantés H, Arreola-Ornelas H, Méndez O. Tendencias de la mortalidad por cáncer de mama en México, 1979-2006. Documento de trabajo. México: Fundación Mexicana para la Salud, 2008. 
La evidencia que se presenta en este trabajo confirma la permanencia de las disparidades en cuanto a la mortalidad por cáncer de mama en la mujer al interior del país. Aun cuando el número de muertes por esta causa se incrementa de forma gradual a lo largo del tiempo a nivel nacional, en algunas entidades del país este aumento es notablemente significativo.

Los principales hallazgos de este trabajo muestran que la tendencia de mortalidad por cáncer de mama ha sido ascendente en el plano nacional durante el periodo de 1980 a 2009, y su distribución regional exhibe, al igual que en los países desarrollados, diferencias norte-sur que se evidencian en una mortalidad ascendente en todas las entidades del país, pero con una distribución heterogénea en la que los estados del norte mantienen las tasas más elevadas. No obstante, hay incrementos considerables de la mortalidad por esta causa en las entidades del centro y del sur del país, aunque éstos se siguen manteniendo por debajo de los estados del norte, lo que puede estar relacionado con la etapa de su transición demográfica.

La idea de que la mortalidad por cáncer de mama se ha asociado con poblaciones de áreas urbanas y más desarrolladas ha cambiado, puesto que ésta ha transitado hacia áreas menos urbanas y menos desarrolladas, como lo muestran los resultados obtenidos en este y otros trabajos. ${ }^{18,19}$ La tendencia ascendente generalizada de la mortalidad por cáncer de mama podría deberse, entre otros factores, a las carencias de infraestructura para el tamizaje y tratamiento, dando como resultado las dificultades en cuanto a detección y manejo oportuno de esta neoplasia en México. ${ }^{20}$

Las poblaciones marginadas en términos sociales, económicos y geográficos tienen mayor probabilidad de morir por algún tipo de cáncer que es prevenible, situación que puede atribuirse, entre otras causas, a la falta de un diagnóstico oportuno y, por consiguiente, a un tratamiento tardío. ${ }^{21}$

\section{Conclusiones}

Los resultados muestran la necesidad de que los distintos sectores de gobierno trabajen de manera conjunta para revertir los niveles de mortalidad por cáncer de mama, educando a la población para su cuidado y prevención con respecto a ésta y a otras enfermedades crónicas, así como para el mejoramiento de la infraestructura y la cobertura de los servicios de salud para una detección oportuna, lo que significa implementar programas adecuados para optimizar la eficiencia, con una distribución regional de los servicios que considere las brechas entre grupos poblacionales del país.
El conocimiento de las enfermedades como causales de muerte y la transformación que tienen en el tiempo son recursos fundamentales para planear estrategias que ayuden a la toma de decisiones y al diseño y ejecución de programas de prevención y educación para la población, enfocados hacia grupos de edad vulnerables, estratos sociales y regiones geográficas, considerando que éstos se relacionan con las transiciones demográfica y epidemiológica, y con diferentes ámbitos que determinan los estilos de vida (biológicos, ambientales, geográficos, culturales, socioeconómicos, entre otros).

El reto es conjuntar esfuerzos para educar a la población hacia el autocuidado de la salud, promoviendo estilos de vida saludables, además de mejorar los sistemas de información y la infraestructura diagnóstica para contribuir a la detección oportuna y al tratamiento adecuado.22

Declaración de conflicto de intereses: Los autores declararon no tener conflicto de intereses.

\section{Referencias}

I. López-Ríos O, Lazcano-Ponce CE, Tovar-Guzmán V, Hernández-Ávila M. La epidemia de cáncer de mama en México. ¿Consecuencia de la transición demográfica? Salud Publica de Mex 1997; 19:259-265. 2. De la Vara-Salazar E, Palacio-Mejía LS, Oropeza C, Hernández M, Lazcano-Ponce E. Perfil epidemiológico de la mortalidad en el sureste de México, 1980-2008. México: INSP, 20II.

3. Caire-Juvera G, Saucedo-Tamayo S, Esparza-Romero J. Mortalidad por cáncer mamario como etapa inicial para el estudio de factores de riesgo nutricios en mujeres de Sonora. [Consultado 20I I agosto].

Disponible en: http://www.ciad.mx/boletin/mayjun05/Cancer_Mamario.pdf 4. Organización Mundial de la Salud. OMS Nota descriptiva No, 297: Cáncer. Febrero de 201 I. [Consultado 20II julio]. Disponible en: http:// www.who.int/topics/cancer/breastcancer/es/index.html

5. Organización Mundial de la Salud. Carga Mundial de Morbilidad, 2004. Ginebra, 2008. [Consultado 201 I julio].Disponible en: http://www.who.int/ healthinfo/global_burden_disease/GBD_report_2004update_full.pdf 6. Mathers CD, Lopez AD, Murray CJL. The burden of disease and mortality by condition: data, methods, and results for 200I. New York: Oxford University Press, 2006:45-93.

7. IARC. World cancer report 2008. Lyon: International Agency for Research on Cancer, 2008.

8. Lacey JV Jr, Kreimer AR, Buys SS, Marcus PM, Chang SC, Leitzmann MF, et al. Breast cancer epidemiology according to recognized breast cancer risk factors in the Prostate, Lung, Colorectal and Ovarian (PLCO) Cancer Screening Trial Cohort. BMC Cancer 2009;9: 84.

9. Peto J. Cancer epidemiology in the last century and the next decade. Nature 2001;41 I:390-395.

10. García-López J E, Gloria-Hernández LE. Mortalidad por causas y ganancias de vida en los últimos veinticinco años. En: La situación demográfica de México, México, 2006:27. [Consultado 201I agosto]. Disponible en: http://www.conapo.gob.mx/publicaciones/sdm2006/ sdm06_03.pdf.

II. Aguayo-Alcaráz G, Jimènez-Pérez LM, Celis-de la Rosa A, CanalesMuñoz JL. Mortalidad por cáncer de mama. Tendencia y distribución geográfica, I98I-200I. Rev Med Inst Mex Seguro Soc 2008;46(4):367-374. 
12. Palacio-Mejía LS, Lazcano-Ponce E,Allen-Leigh B, Hernández-Ávila M. Diferencias regionales en la mortalidad por cáncer de mama y cérvix en México entre 1979 y 2006. Salud Publica Mex 2009;5I supl 2:S208-S2I 9. 13. Knaul FM, Nigenda G, Lozano R,Arreola-Ornelas H, Langer A, Frenk J. Cáncer de mama en México: una prioridad apremiante. Salud Publica Mex 2009;5 I supl 2:S335-S344

14. Dirección General de Información en Salud (DGIS). Base de datos de defunciones 1979-2009 [en línea]: Sistema Nacional de Información en Salud (SINAIS). México: Secretaría de Salud. [Consultado 20II abril]. Disponible en: http://www.sinais.salud.gob.mx

15. Consejo Nacional de Población. Proyecciones de la población de México, 1980-2030, 2005-2050. México. CONAPO.

16. Organización de las Naciones Unidas. División de Población del Departamento de Asuntos Sociales y Económicos de la Secretaría de Naciones Unidas. En: Perspectivas de la Población Mundial: Revisión 2008 y Perspectivas de la Urbanización Mundial: Revisión 2003. [Consultado 201 I abril 4]. Disponible en: http://esa.un.org/unpp

17. Welti C, ed. Demografía II. México: Programa Latinoamericano de Actividades en Población (PROLAP). México: Instituto de Investigaciones Sociales (IIS/UNAM), 1998:36-44, 60-66.
18. Parkin DM, Bray FI, Devesa SS. Cancer burden in the year 2000. The global picture. Eur J Cancer 200I; 37(Suppl. 8): S4-S66.

19. López-Carrillo L, Suárez-López L. Cáncer de mama. En: ChávezGalindo A, Uribe-Zúñiga P, Núñez-Fernández L, Palma-Cabrera Y, (eds). La salud reproductiva en México.Análisis de la encuesta nacional de salud reproductiva 2003. México: Secretaría de Salud/Centro Regional de Investigaciones Multidisciplinarias, UNAM, 2007: 236-252.

20. López-Carrillo L, Suárez-López L,Torres-Sánchez L. Detección del cáncer de mama en México: síntesis de los resultados de la encuesta nacional de salud reproductiva. Salud Publica Mex 2009; 5 I Supl. 2: S345-S349.

21 . Hairon N. Report finds inequity in access to breast surgery services. Nurs Times 2008;104(II):2I-22.

22. González-Robledo LM, González-Robledo MC, Nigenda G, LópezCarrillo $L$.Acciones gubernamentales para la detección temprana del cáncer de mama en América Latina. Retos a futuro. Salud Publica Mex 2010;52:533-543. 